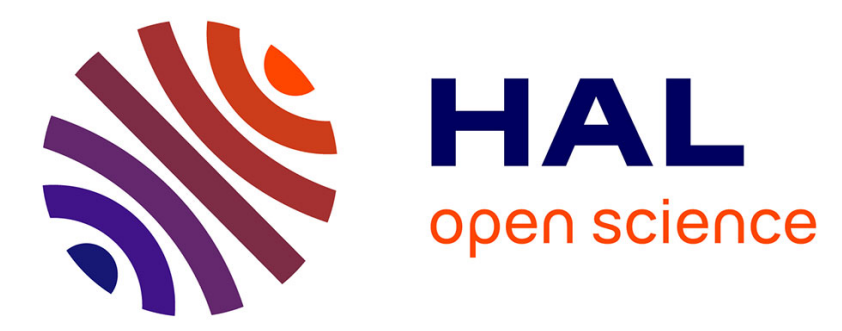

\title{
A wavelet-based method for local phase extraction from a multi-frequency oscillatory signal.
}

\author{
Stéphane G. Roux, Tristan Cenier, Samuel Garcia, Philippe Litaudon,
} Nathalie Buonviso

\section{- To cite this version:}

Stéphane G. Roux, Tristan Cenier, Samuel Garcia, Philippe Litaudon, Nathalie Buonviso. A waveletbased method for local phase extraction from a multi-frequency oscillatory signal.. Journal of Neuroscience Methods, 2007, 160 (1), pp.135-43. 10.1016/j.jneumeth.2006.09.001 . hal-00173653

\section{HAL Id: hal-00173653 \\ https://hal.science/hal-00173653}

Submitted on 20 Sep 2007

HAL is a multi-disciplinary open access archive for the deposit and dissemination of scientific research documents, whether they are published or not. The documents may come from teaching and research institutions in France or abroad, or from public or private research centers.
L'archive ouverte pluridisciplinaire HAL, est destinée au dépôt et à la diffusion de documents scientifiques de niveau recherche, publiés ou non, émanant des établissements d'enseignement et de recherche français ou étrangers, des laboratoires publics ou privés. 
A wavelet-based method for local phase extraction from a multi-frequency oscillatory signal

Roux Stéphane G. ${ }^{\mathrm{a}}$, Cenier Tristan ${ }^{\mathrm{b}}$, Garcia Samuel ${ }^{\mathrm{b}}$, Litaudon Philippe ${ }^{\mathrm{b}}$ and Buonviso Nathalie ${ }^{b, *}$

${ }^{a}$ Laboratoire de Physique, Ecole Normale Supérieure de Lyon, UMR 5672, 46 allée d'Italie, 69364 Lyon cedex 07, France.

${ }^{\mathrm{b}}$ Neurosciences \& Systèmes Sensoriels, Université Lyon 1-CNRS, UMR 5020, 50 avenue Tony Garnier, 69366 Lyon cedex 07, France. IFR 19, Institut Fédératif des Neurosciences de Lyon.

Number of text pages: 23

Number of figures: 7

Number of tables: 0

*Corresponding author:

Nathalie BUONVISO, Neurosciences \& Systèmes Sensoriels, Université Lyon 1 - CNRS, 50 avenue Tony Garnier, 69366 Lyon cedex 07, France

Tel.: $33(0) 437287461$

Fax: $33(0) 437287601$

e-mail: buonviso@olfac.univ-lyon1.fr 


\begin{abstract}
One of the challenges in analyzing neuronal activity is to correlate discrete signal, such as action potentials with a signal having a continuous waveform such as oscillating local field potentials (LFPs). Studies in several systems have shown that some aspects of information coding involve characteristics that intertwine both signals. An action potential is a fast transitory phenomenon that occurs at high frequencies whereas a LFP is a low frequency phenomenon. The study of correlations between these signals requires a good estimation of both instantaneous phase and instantaneous frequency. To extract the instantaneous phase, common techniques rely on the Hilbert transform performed on a filtered signal, which discards temporal information. Therefore, time-frequency methods are best fitted for nonstationary signals, since they preserve both time and frequency information. We propose a new algorithmic procedure that uses wavelet transform and ridge extraction for signals that contain one or more oscillatory frequencies and whose oscillatory frequencies may shift as a function of time. This procedure provides estimates of phase, frequency and temporal features. It can be automated, produces manageable amounts of data and allows for human supervision. Because of such advantages, this method is particularly suitable for analyzing synchronization between LFPs and unitary events.
\end{abstract}

\title{
Keywords
}

Rat, olfaction, wavelet transform, ridge extraction, oscillation phase 


\section{Introduction}

For about ten years, the study of local field potentials (LFPs) has received an increasing interest, particularly because such signals appear to be relevant indicators of information processing. LFPs, which are considered as the summation of excitatory and inhibitory dendritic potentials (Mitzdorf, 1987), are often oscillatory. Oscillatory synchrony of LFPs between different cortical areas probably has a true functional role. Indeed, it has been shown in human intra-cranial recordings that the holding of visual information in short-term memory is accompanied by oscillatory synchrony in the $\beta$ band $(15-20 \mathrm{~Hz})$ across distinct visual areas (Tallon-Baudry et al., 2001). In a similar experiment in monkeys, two sites located over the posterior infero-temporal cortex are synchronized in the $\beta$ band during a memory maintenance task in correct trials, while the synchrony fails to develop in incorrect trials (Tallon-Baudry et al., 2004). On the other hand, as LFPs oscillations are supposed to originate in the rhythmical synchronization of groups of neurons (Mitzdorf, 1987), several teams have studied the temporal relationship existing between oscillations and neuronal spike discharges. It has thus been reported that both activities can become phase-locked under certain behavioral or perceptual conditions (Murthy and Fetz, 1996; Fries et al., 2001; Siegel and König, 2003). Hence, when studying the coherence between LFPs oscillations from different brain regions, or the synchronization between spikes and LFPs oscillations, the quantification of oscillation phase becomes crucial and the results will depend on its accuracy.

The traditional Hilbert transform method for phase extraction can only be applied after the signal has been Fourier-filtered around the frequency band of interest if the signal contains oscillations at different frequencies. Although this method is very efficient, it has a major drawback as it suppresses all temporal information. Indeed, the Fourier representation describes the signal as a sum of infinite oscillations and mixes time and frequency information. For non-stationary signal studies, many time-frequency methods exist that analyze the local frequency composition of the signal while preserving temporal information 
(see Boashash, 1992a,b for review). Among these methods, parametric methods require the operator to have an insight into the data: specifically, the operator needs to determine the frequency range of the oscillatory phenomenon and/or the time boundaries of the oscillatory epochs. Conversely, non-parametric methods such as time-frequency representations (Flandrin, 1993) offer a convenient setup, in which the problem of local amplitude estimation is well understood and addressed, but only in the case of single component signals (Boashash, 1992a,b; Delprat et al., 1992). Further refinements of this setup, described by Carmona et al. (1997), can be used to address multi-component or noisy signal (Carmona et al., 1999). However, an important issue is the computational cost of such methods. The time-frequency map computation multiplies the original amount of data several folds, which could rapidly saturate the computational capabilities of any computer, rendering any further processing or human visual check virtually impossible. This is particularly true in a situation where a high sampling rate leads to a huge number of samples and where, for each sample, precise phase and frequency information are to be extracted.

Here we propose a new algorithmic procedure, based on wavelet ridge extraction (Delprat et al., 1992), to extract instantaneous frequency and instantaneous phase information from high sample rate signals. This method is very robust even when multiple oscillatory regimes are simultaneously present. Moreover, it produces a computationally manageable amount of data. Consequently, it is well suited for the study of synchronization between spike activity and LFPs oscillations in the olfactory system of the freely breathing rat, where LFPs oscillate in least 2 frequency bands, $\beta$ and $\gamma$, both regimes alternating within each respiratory cycle (Buonviso et al., 2003).

\section{Methods}

\section{Continuous wavelet transform and wavelet ridge}




\section{Continuous wavelet transform}

In order to preserve both time and frequency information, one commonly uses a timefrequency representation based on a Windowed Fourier Transform (WFT) or a Continuous Wavelet Transform (CWT). We chose to use the CWT instead of the WFT because the window size depends on the screened frequency, in the case of CWT, as opposed to the WFT fixed window size. This represents an asset of the method since the duration of oscillations often shortens as the frequency increases. CWT provides a better compromise between time and frequency resolution. The CWT yields a series of coefficients in time representing the evolution of the frequency content (Mallat, 1998) of the signal $x$ by:

$$
T_{\Psi}[x](t, a)=\int x(t) \Psi_{t, a}^{*}(s) d s \quad a>0, t \in R
$$

where $t$ stands for time, $a$ for the scale and $*$ for the complex conjugate. The functions $\Psi_{\mathrm{t}, \mathrm{a}}$ are obtained by dilation and translation of a unique waveform $\Psi: \Psi(t, a)=\left(\frac{1}{\sqrt{a}}\right) \Psi\left(\frac{s-t}{a}\right)$. The function $\Psi$, called mother wavelet, is a function with mean value equal to zero, and is characterized by its center frequency $\left(\mathrm{f}_{0}\right)$, its spread in time $\sigma_{t}=\int|\Psi(s)|^{2} d s$ and its spread in frequency $\sigma_{f}=\int|\hat{\Psi}(\omega)|^{2} d \omega$ (where $\hat{\Psi}$ indicates the Fourier transform). By decreasing or increasing $a$, the basis function $\Psi_{t, a}$ is fitted to a segment of $x(t)$; hence, $a$ indirectly represents the frequency of the signal. Squaring the results and dividing by the scale $P_{x}(t, a)=\frac{\left|T_{x}(t, a)\right|^{2}}{a}$ generates a time-scale energy density distribution called normalized scalogram. $P_{x}(t, a)$ represents the energy of the signal in a time-frequency box whose center and size are defined by $\left(t, \frac{f_{0}}{a}\right)$ and $\left(a \sigma_{t}, \frac{\sigma_{f}}{a}\right)$ respectively : when $f\left(=\frac{f_{0}}{a}\right)$ increases ( $a$ decreases), the time resolution improves and the frequency resolution worsens. 
Different families of mother wavelets can be applied. The choice is influenced by the nature of the information to be extracted. For the determination of instantaneous frequency, the most commonly used wavelet is the so-called Morlet wavelet (Kronland-Martinet et al. 1987), defined in the time domain by $\Psi(t)=\frac{1}{2 \pi} \cdot e^{-i 2 \pi f_{0}} e^{-t^{2} / 2}$ and in the frequency domain by $\hat{\Psi}(f)=\frac{1}{2 \pi} e^{-2 \pi^{2}\left(f-f_{0}\right)^{2}}$ A wavelet family is characterized by the constant $\omega_{0}=2 \pi f_{0}$. For large $\omega_{0}$, frequency resolution improves at the expense of time resolution. To obtain a wavelet with mean value equal to zero, we need to set $\omega_{0}>5$ (Grossmann et al., 1989).

\section{Wavelet ridge extraction}

The method determining instantaneous frequency from wavelet ridges was first proposed by Delprat et al. (1992) where the phase coherence of the wavelet transform was used to obtain a numerical estimate of the ridge. For noisy, and/or multi-component signals, Carmona et al. $(1997,1999)$ proposed to use the localization of the scalogram maxima instead. Note that the detection algorithm is only a particular post-processing method of a time-frequency transform. It thus can be used with other time-frequency energy representations such as WFT or more generally the family of smoothed Wigner distributions (Auger et al., 1995, Carmona et al., 1999).

Considering a sinusoidal signal given by the complex exponential function: $x(t)=e^{-i 2 \pi f_{T} t}$ where $f_{T}$ denotes the frequency, the wavelet transform of the signal is: $T_{\Psi}[x](t, a)=\sqrt{a} \hat{\Psi}\left(a f_{T}\right) e^{-i 2 \pi f_{T} t}$

Substituting the Fourier transform of the Morlet wavelet into this equation, we obtain for the normalized scalogram $P_{x}(a, b)=\frac{1}{4 \pi^{2}} e^{-2 \pi^{2}\left(a f_{T}-f_{0}\right)^{2}}$. 
Deriving this scalogram with respect to $a$, we obtain $\frac{\delta P_{x}}{\delta a}(a, b)=f_{T}\left(a f_{T}-f_{0}\right) e^{-2 \pi^{2}\left(a f_{T}-f_{0}\right)^{2}}$. In such conditions, the point $\left(t, a_{R}\right)$ where $\frac{\delta P_{x}}{\delta a}=0$ verifies $a_{R} f_{T}-f_{0}=0$ and corresponds to the maximum energy of the scalogram. The scalogram is then essentially maximum in the neighborhood of a curve $a_{R}(t)$, which is the ridge of the wavelet transform, related to the instantaneous frequency of the signal by the simple expression $\frac{f_{0}}{a_{R}}=f_{T}$. If the scalogram is defined in terms of frequency instead of scale, i.e. $P_{x}(f, b)$ where $f=\frac{f_{0}}{a}$, the instantaneous frequency can be read directly from the spectral location of the ridge.

In this paper, we use the localization of scalogram maxima to collect both instantaneous frequency and instantaneous phase information. Indeed, using a complex wavelet allows us to define the instantaneous phase of the oscillation mode simply by the angle of the wavelet transform, $\arg \left(T_{x}\left(a_{R}, b\right)\right)$ along the corresponding ridges. This method has proven efficient even on noisy signals, but it is not suitable for long sets of data since it can prove too time consuming, and produces exceedingly large data files. We propose here a new implementation based on two layers of processing, optimized for data with high sampling frequency.

\section{Implementation for high-sampled signals}

Our goal was to create tools that would allow the comparison of temporal aspects of two components in a signal, where the components are different in nature and characteristics. One component is a fast transitory signal (i.e. neuronal spikes), occurring at high frequencies, thus demanding a high sampling frequency $\left(f_{s}\right)$. The other component (i.e. LFPs) is slow and intermittent and exists in mid-range frequencies (we set the range as $\left[f_{\min } ; f_{\max }\right]$ ); therefore a low sampling rate $\left(2 f_{\max }\right)$ is enough for this component. From the low frequency events, we 
wanted to extract precise temporal position, instantaneous frequency and instantaneous phase. Furthermore we wanted our method to be as automated as possible. Since we needed to correlate this instantaneous phase with the high frequency events, we designed a two layers processing. First, we computed a low time-resolution scalogram (LTRS) from the filtered and under-sampled raw signal (from $f_{s}$ to $2 f_{\max }$ ) to obtain a coarse estimation of the oscillations positions. Second, we computed scalograms from the raw signal, around the coordinates of the LTRS maxima. These high time-resolution scalograms (HTRS) can be used to extract accurate phase and frequency information from each recorded sample. This method preserves high-resolution information where needed, which is crucial for exploiting the data, and reduces the overall amount of data, thus facilitating data manipulation and allowing for human supervision.

\section{1- Low time-resolution wavelet transform for mode localization (Fig. 1A)}

When looking for oscillations in a $0-f_{\max }$ range, we filtered the signal between $0-2 f_{\max }$, and then sampled it at $2 f_{\max }$. Then the wavelet transform was performed on this smoothed undersampled signal to obtain a LTRS from which the coordinates in time and frequency of all local maxima above a given threshold were extracted (Fig. 1A, circle). The positions of the maxima were a coarse estimate of the position of the amplitude maxima of oscillatory episodes present in the signal. One asset of this method is that, when under-sampled, the signal is dramatically smoothed, thus enhancing the signal to noise ratio. This, combined with the use of a threshold, safely removes the maverick maxima.

\section{2- Ridge extraction from high-resolution scalogram (Fig. 1B)}

From the coarse estimation of the position of the maxima obtained from the LTRS, $\left(t \pm \frac{1}{2 f_{\max }}\right)$, we computed the HTRS from the raw signal in the neighborhood of this position 
(i.e. for each time sample around the coarse maximum) in order to find the exact location of the maxima $\left(t \pm \frac{1}{f_{s}}\right)$. From this precise location, the wavelet ridge was computed recursively: from each ridge point, the next one was defined as the maximum of the wavelet transform computed at the next sample time in neighboring frequencies. The ridge stopped when a maximum with energy below a threshold was detected. The ridge computation was performed both forwards and backwards from each maximum. For each maximum detected on the LTRS we obtained a wavelet ridge and its starting and ending times. Then, for each point of that ridge we obtained the local oscillating regime characteristics: instantaneous frequency and instantaneous phase. All computed ridges were then pasted on the LTRS in order to visually check the data computed both on high and low time-resolution.

All programs and routines were written and implemented in $\mathrm{C}$ language and Matlab (Mathworks).

\section{Example of application on a biological signal: odor-evoked activity in the mammalian}

\section{olfactory system}

Experiments and procedures have been previously explained in detail (Buonviso et al., 2003; Litaudon et al., 2003). Briefly, preparation and recordings were carried out in naturally breathing, anesthetized Wistar rats. Anesthesia (urethane $1.5 \mathrm{~g} / \mathrm{kg}$ ) was maintained by supplemental doses when necessary. All surgical procedures were conducted in strict accordance with the European Council Guidelines. Electrophysiological activity was collected from the olfactory bulb and piriform cortex using glass micropipettes. Extracellular activity was recorded as a broadband signal $(0.1 \mathrm{~Hz}$ to $5 \mathrm{kHz})$, amplified and sampled at $10 \mathrm{kHz}\left(f_{s}\right)$ in order to obtain a temporal resolution well suited for both spikes and LFPs analysis. The signal was then acquired on a personal computer using the IOTech acquisition system (WaveBook). A recording session lasted $15 \mathrm{sec}$, distributed as follows: $5 \mathrm{sec}$ of spontaneous activity (or pre- 
stimulus period), $5 \mathrm{sec}$ of odor-evoked activity and $5 \mathrm{sec}$ of activity after stimulation. The signal being sampled at $10 \mathrm{KHz}$, each file contained 150,000 samples. The single unit activity was extracted from the broadband signal by band-passing from 300 to $3000 \mathrm{~Hz}$ (FFT Blackman filter, Matlab software). The spikes were extracted from the signal and individually time stamped. Instantaneous phase and frequency of oscillatory events were measured with the wavelet ridge extraction method described above. In the olfactory system, oscillatory components range from 10 to $80 \mathrm{~Hz}$ (Buonviso et al., 2003) and more specifically in two frequency bands defined as $\beta(10-35 \mathrm{~Hz})$ and $\gamma(35-80 \mathrm{~Hz})$. Therefore, the low-resolution wavelet transform was calculated using $f_{\max }=100 \mathrm{~Hz}$ and $\omega_{0}=7$. Since the data of biological significance was the difference in oscillatory activity between spontaneous and odor-evoked activity, a fixed threshold was defined as mean \pm 5 standard deviations of the time-frequency energy contained in the pre-stimulus period. Extracted ridges were sorted as belonging to the $\beta$ or $\gamma$ band according to the frequency of their maximum. Doing this, we characterized onset and duration of each oscillatory epoch, in both bands, and instantaneous phase and frequency along the ridge. Then, for each time-stamped spike that occurred during an oscillatory epoch, we spotted the instantaneous phase of the LFPs signal at that time stamp and then built two spike preferential instantaneous phase histograms (SPIPH), one for each band ( $\beta$ or $\gamma)$.

\section{Results}

\section{Testing of the method on a synthetic signal}

The reliability of the method for instantaneous frequency extraction is well documented (Delprat et al., 1992, Carmona et al., 1997). In this study, we tested this method for instantaneous phase estimation in a synthetic signal and compared the results with those obtained with the commonly used Hilbert method (Fig. 2). The test signal contains two oscillatory components:

$A_{i} \exp \left(-\left(t-t_{i}\right)^{2} / 2 \sigma_{i}^{2}\right) \sin (2 \pi \phi(t))$ with for $\mathrm{i}=1, A_{1}=1.5, \sigma_{l}=1, t_{1}=2.5$ for $2<t<3$ and 
for $\mathrm{i}=2, A_{2}=1, \sigma_{2}=1.5, t_{2}=2$ for $0.5<t<4.5$

The local frequencies are defined by $f_{i}(t)=\frac{d \phi}{d t}$ with $f_{\text {theo1 }}(t)=f_{1}+40 *\left(t-t_{1}\right)$ with $f_{1}=30$ and $f_{\text {theo } 2}(t)=f_{2}-4 \cos (4 t)$ with $f_{2}=20$. Both modes present a frequency shift and are not permanent (Fig. 2A). The signal was sampled at $10 \mathrm{kHz}$ and a Gaussian white noise was added. Scalogram in figure $2 \mathrm{~B}$ shows both the wavelet transform of this signal and the extracted ridges. It is clear that both frequency modes are well distinguished and the positions of the ridges are accurately detected. Thus, their respective phase is very well estimated at any time. Indeed, theoretical and calculated phases are perfectly superimposed for both components (Fig. 2C, D). It is noteworthy that both onset and offset of each oscillating event have been automatically detected. In contrast, the extraction of the phase using the angle of Hilbert transform requires that the signal be filtered a priori and the filter be carefully chosen. In this example, the signal has been filtered between $10-28 \mathrm{~Hz}$ and $28-50 \mathrm{~Hz}$. The graphs in figure 2 (E, F) reveal that, for $t>2.3$, the components were not well separated and gave an unsatisfactory estimation of the phase. Notice that if we had chosen an optimal filter to separate both modes, the phase estimate would have been quite good; nevertheless, it would still have been at the expense of temporal and frequency information. This is illustrated in figure $2 \mathrm{~F}$ where the method finds a phase for $\mathrm{t}<2$ even when the signal is not oscillating. Therefore, in this case, the phase extracted from the Hilbert transform should only be used on portions of the signal (oscillatory periods), after manually setting the limits. In contrast, our method is frequency shift tolerant, and the use of a time-frequency representation allows us to keep both precise temporal and frequency information.

The automation allows us to perform the analysis of frequency and phase estimation versus signal to noise ratio. In order to test to what extent our method is noise-resistant, we performed 100 realizations of the signal for different signal to noise ratios. Signal to noise 
ratio is defined as $S N R=\left(\frac{A_{s}}{A_{n}}\right)^{2}$ where $A_{s}$ and $A_{n}$ are respectively the root mean square amplitude of the signal and the noise. For each realization we obtained a set of wavelet ridges. The correct ridges were easily identified as the two longest or most energetic ones. Both ridges gave us an estimation of the frequency $f(t)$ and phase $\phi(t)$. Then we computed, for the frequency, the normalized bias and standard deviation: $<f(t)-f_{\text {theo }}>/ f_{\text {theo }}$ and $<\hat{f}(t)^{2}>-<\hat{f}(t)>^{2}$ and, for the phase, the bias and standard deviation $<\hat{\phi}(t)-\phi_{\text {theo }}>$ and $<\hat{\phi}(t)^{2}>-<\hat{\phi}(t)>^{2}$. The results are shown in figure 3 . The normalized bias for the frequency and the bias for the phase are very small and stay constant below $10^{-2}$ even with very low SNR. Note that this SNR is computed at the frequency sampling. As we apply the filtering/under-sampling process, the SNR increases as the square root of the under-sampling rate. The standard deviation behaves as $1 / \sqrt{S N R}$.

\section{Limitations of the method}

There are a few limitations to the extent to which this method can be applied. One is intrinsic to the wavelet ridge extraction method and is encountered when some components of the signal cross each other in frequency domain. Indeed, if two instantaneous frequencies cross each other in the time-frequency plane, their separation is not unique in general cases (Boashash, 1992a). Figure 4A shows a signal holding two oscillatory components. As evidenced in figure 4B, the ridges extracted from this signal merge: it is then impossible to decide which ridge after the crossing point is the continuation of which ridge before that point.

Another limit depends on the choice of the parameter $\omega_{0}$ of the wavelet. This choice will depend on the frequency composition of the signal. This factor sets the shape of the wavelet. 
When $\omega_{0}$ is set too low (Fig. 5B), the scalogram looses frequency resolution, and it is impossible to distinguish between two components of the signal, especially if they exist in close frequency ranges. When $\omega_{0}$ is set too high (Fig. 5D), the time-resolution of the scalogram is too poor. To address this problem, a simple visual check of the LTRS allows us to judge the validity of the chosen $\omega_{0}$ value (Fig. 5C). Once the correct value is set, full scale automation becomes possible.

Finally, although the method is very noise resistant, too much noise could increase the number of detected maxima, thus a ridge might falsely be detected several times. In our case, the filtering and under-sampling smoothed the signal enough to avoid maverick maxima. In other cases, an additional data sorting routine must be implemented to remove redundant ridges. The visual information given by the LTRS and the pasting of the ridges allow us to visually check the validity of the sorting step.

\section{Example of application on a biological signal: odor-evoked activity in the mammalian} olfactory system

As mentioned above, it is an emerging concept in neuroscience that the temporal aspects of brain signals, such as relations between oscillatory LFPs and single neuron activity, could play a major part in the representation of complex information. The custom wavelet ridge extraction method presented here was originally designed to study such temporal aspects, but can be extended to any other subject fields.

As previously described (Buonviso et al., 2003, Neville and Haberly, 2003), odor-evoked activity in the mammalian olfactory system presents oscillatory bursts in both bulbar and cortical LFPs (Fig. 6, 7). Such activity exhibits 2 frequency bands ( $\beta$ and $\gamma$ activities, respectively $15-35 \mathrm{~Hz}$ and $35-80 \mathrm{~Hz}$ ) that alternate along a single respiratory cycle. The spiking activity in these structures is not linear: cells tend to fire preferentially during oscillatory periods. Here, the qualitative and quantitative study of spike phase-locking 
requires phase measurements to be conducted with the same accuracy in every frequency band, while preserving temporal information. The wavelet ridge extraction method meets all these requirements. It was performed as described in the methods section; on average, analysis of a $15 \mathrm{sec}$ recording (sampling rate: $10 \mathrm{kHz}$ ) took about 21 seconds. It successfully extracted the temporal information and instantaneous frequency and phase of the oscillatory bursts, even when two oscillatory modes were present, from both bulbar and cortical structures (Fig. 6A to 6E, Fig. 7A, B). The extracted phases are linear for all ridges, as can be seen in Fig. 6E and 7B.

Then, knowing i) the onset and duration of each oscillatory period, ii) the instantaneous phase and frequency of the signal at every time-stamp (or phases if more than one oscillatory events exist at a given time-stamp) and iii) the time-stamp of every spike, we were able to construct spike preferential instantaneous phase histograms (SPIPHs) for each frequency band. Examples of typical SPIPH are presented in figures 6 and 7. In the olfactory bulb (Fig.6F, G), the histograms for a particular neuron reveal the tendency of that neuron to spike preferentially during the falling phase of the LFP $\gamma$ oscillations. In the piriform cortex, the single-trial SPIPH (Fig. 7C) suggests the same tendency: spikes occur at a preferential phase during $\gamma$ epochs. To verify this tendency we constructed an average SPIPH with data collected from 6 cells (Fig 7D). The phase locking between spikes and LFPs thus appeared very clearly and was quantified (mean phase $=-3.00 \pm 1.13 \mathrm{rad}$ ). Its significance was estimated using the Rayleigh circular directionality test $(\mathrm{p}<0.001)$.

\section{Discussion}

Study of local field potentials could be a valuable tool toward understanding brain functions. Many studies focus on trying to elucidate the role and mechanisms of LFPs. Depending on the experimental conditions, the recorded signals may possess different characteristics, i) stationary or non-stationary ii) containing one or more oscillatory frequencies iii) oscillatory 
frequencies may shift as a function of time. From such signals, the important information to be accurately extracted are local frequency and phase of each oscillatory epoch as well as its temporal characteristics (onset and duration). In this paper, we described a method using ridge extraction on CWT that answers all these requirements.

The first stage of the method consists of extracting the spectral components of the signal. CWT method has been chosen because of its lesser susceptibility to non-stationarity (vs Hilbert method) and because of its better time-frequency resolution (vs FWT method). Indeed, traditional signal processing methods that rely on the Hilbert transform of a filtered signal are well adapted for signals that exhibit permanent oscillation. But, in the case where the oscillations appear intermittently, these methods fail to give information either on time or on frequency characteristics of the oscillations. As shown in figure 2, Hilbert transform failed to detect boundaries of oscillatory events and erroneously found a phase where the signal did not appear to oscillate. For such non-stationary signals, non-parametric time-frequency methods (Flandrin, 1993) offer a more convenient setup for conducting research, without requiring any a priori knowledge of the recorded data. In this type of representation, a correct estimate of the signal frequency composition depends on the choice of the window size. The CWT has been preferred to the widely spread WFT due to CWT's ability to detect oscillations with very different frequencies and durations. This ability comes from the fact that in CWT the window size varies with the inverse of the frequency in opposition to the WFT where the window size is constant. As the duration of the window becomes shorter for higher-frequency bands, CWT provides a better compromise between time and frequency resolutions. CWT is characterized by the parameter $\omega_{0}$ which should be set to optimize temporal and frequency resolution. For frequencies of interest ranging from 15 to $100 \mathrm{~Hz}$, a value around 7 was commonly used (Tallon-Baudry et al., 1997; Ravel et al., 2003).

From this time-frequency representation, instantaneous phase and frequency information was obtained using the ridge extraction method which presents several advantages. First, the two- 
layer algorithm reduces computation time as it only focuses on self-defined areas of interest. It considerably reduces the amount of stored data without impairing the ability to check the validity of the results. Second, this method is very noise resistant as shown in figure 3 . Moreover, the use of both a smoothing filter and a threshold for detecting maxima prevents multiple detections of the same ridge. As mentioned above, this method proved unable to follow the frequencies of multiple oscillatory components when these components' frequencies crossed each other (see figure 4). Nevertheless, this limitation, which is inherent to time-frequency representation, is not troublesome for analyzing neuronal activity where oscillatory events are generally well separated both in time and frequency. One more asset of the proposed method is that it can be fully automated after a few preliminary tests.

As previously underlined, accurate estimation of instantaneous phase and frequency of oscillatory activity is a prerequisite to the analysis of temporal relationship between neuronal spike discharges and oscillations. In the olfactory system, the temporal relationship between spikes and LFPs have been approached in several studies conducted on insect (Laurent et al., 1996), fish (Friedrich et al., 2004), and rabbit (Kashiwadani et al., 1999). All of them used an a priori signal filtering in a precise frequency band, and then extracted peaks, troughs and zero-crossings of the LFPs signal to use as phase reference points. These methods are convenient and valid on data collected in those cases because LFPs oscillate within a single frequency band and the signal is stationary. Such methods cannot be applied to analyze recordings from the freely breathing rat olfactory system, however, where signals are largely non-stationary and exhibit frequency shifts. Indeed, odor-evoked LFPs exhibit periods of $\beta$ and $\gamma$ oscillatory activity that alternate during a respiratory cycle (Buonviso et al., 2003; Neville and Haberly, 2003), but seldom overlap during very short periods, and the frequency often changes during $\beta$ episodes. Our wavelet ridge extraction method overcomes the extracomplexity of LFPs from freely breathing rat olfactory system. Furthermore, for $\beta$ and $\gamma$ 
oscillations occurring at the same time, time-stamped spikes would yield two phases, one for each ridge or frequency band. The results obtained regarding phase relationship between spikes and LFPs are very promising.

\section{Abbreviations}

LFP local field potential

CWT continuous wavelet transform

WFT windowed Fourier transform

LTRS low time-resolution scalogram

HTRS high time-resolution scalogram

SPIPH spike preferential instantaneous phase histogram

SNR signal to noise ratio

\section{Acknowledgements}

This work was supported by a grant from CNRS "Programme Interdisciplinaire: Cognition et Traitement de l'Information" 


\section{References}

Auger F. Flandrin P. Improving the readability of time-frequency and time-scale representations by the reassignment method. IEEE Trans. Signal Proc., 1995; 43 (5): 10681089.

Boashash B. Estimating and interpreting the instantaneous frequency of a signal. I. Fundamentals. Proc. IEEE, 1992a; 80: 520-39.

Boashash B. Estimating and interpreting the instantaneous frequency of a signal. II. Algorithms and applications. Proc. IEEE, 1992b; 80: 540-68.

Buonviso N, Amat C, Litaudon P, Roux S, Royet JP, Farget V, Sicard G. Rhythm sequence through the olfactory bulb layers during the time window of a respiratory cycle. Eur. J. Neurosci., 2003; 17: 1811-9.

Carmona R, Hwang W, Torresanni B. Characterization of signals by the ridges of their wavelet transform. IEEE Trans. Signal Proc., 1997; 45: 2586-90.

Carmona R, Hwang W, Torresanni B. Multiridge detection and time-frequency reconstruction. IEEE Trans. Signal Proc., 1999; 47: 480-92.

Delprat N, Escudie B, Guillemein P, Kronland-Martinet R, Tchamitchian P, Torresanni B. Assymptotic wavelet and Gabor analysis: extraction of instantaneous frequencies. IEEE Trans. Inform. Theory, 1992; 38: 644-64.

Flandrin P. Temps-fréquence. Traité des nouvelles technologies. Hermès: Paris, 1993: 408.

Friedrich RW, Habermann CJ, Laurent G. Multiplexing using synchrony in the zebrafish olfactory bulb. Nat. Neurosci., 2004; 7: 862-71.

Fries P, Reynolds JH, Rorie AE, Desimone R. Modulation of oscillatory neuronal synchronization by selective visual attention. Science, 2001; 291: 1560-3.

Grossman A, Kronland-Martinet R, Morlet J. Reading and understanding continuous wavelet transform. In Combes JM, Grossman A, Tchamitchian P, editors. Wavelets, time-frequency methods and phase space. Springer-Verlag: Berlin, 1989: 2-20. 
Kashiwadani H, Sasaki YF, Uchida N, Mori K. Synchronized oscillatory discharges of mitral/tufted cells with different molecular ranges in the rabbit olfactory bulb. J. Neurophysiol., 1999; 82: 1786-92.

Kronland-Martinet R, Morlet J, Grossmann A. Analysis of sound patterns through wavelet transforms. Int. J. Patt. Recogn. Art. Intell., 1987; 1: 273-302.

Laurent G, Wehr M, Davidovitz H. Temporal representations of odors in an olfactory network. J. Neurosci., 1996; 16: 3837-47.

Litaudon P, Amat C, Bertrand B, Vigouroux M, Buonviso N. Piriform cortex functional heterogeneity revealed by cellular responses to odours. Eur. J. Neurosci., 2003; 17: 2457-61.

Mallat S. A wavelet tour in signal processing. Academic Press: New York, 1998.

Mitzdorf U. Properties of the evoked potential generators: current source-density analysis of visually evoked potentials in the cat cortex. Int. J. Neurosci., 1987; 33: 33-59.

Murthy VN, Fetz EE. Synchronization of neurons during local field potential oscillations in sensorimotor cortex of awake monkeys. J. Neurophysiol., 1996; 76: 3968-82.

Neville KR, Haberly LB. Beta and gamma oscillations in the olfactory system of the urethane-anexthetized rat. J. Neurophysiol., 2003; 90: 3921-30.

Ravel N, Chabaud P, Martin C, Gaveau V, Hugues E, Tallon-Baudry C, Bertrand O, Gervais R. Olfactory learning modifies the expression of odour-induced oscillatory responses in the gamma (60-90 Hz) and beta (15-40 Hz) bands in the rat olfactory bulb. Eur J Neurosci., 2003, $17: 350-8$

Siegel M, Konig P. A functional gamma-band defined by stimulus-dependent synchronization in area 18 of awake behaving cats. J. Neurosci., 2003; 23: 4251-60.

Tallon-Baudry C, Bertrand O, Delpuech C, Permier J. Oscillatory gamma-band (30-70 Hz) activity induced by a visual search task in humans. J Neurosci., 1997, 17:722-34.

Tallon-Baudry C, Bertrand O, Fischer C. Oscillatory synchrony between human extrastriate areas during visual short-term memory maintenance. J. Neurosci., 2001; 21: RC177 1-5. 
Tallon-Baudry C, Mandon S, Freiwald WA, Kreiter AK. Oscillatory synchrony in the monkey temporal lobe correlates with performance in a visual short-term memory task. Cereb. Cortex, 2004; 14: 713-20. 


\section{Figure legends}

Figure 1: (A) Low temporal resolution scalogram $\left(f_{\max }=100 \mathrm{~Hz}\right)$. The maximum intensity is represented by the circle. In the neighborhood of this maximum (dashed square), we compute the high temporal resolution $\left(f_{s}=10 \mathrm{kHz}\right)$ scalogram (B) to obtain a more precise location of the maximum $(*)$. From this precise location, the wavelet ridge is recursively extracted in the left and right direction by computing only the neighboring frequency at the next sample time represented by the grey coded pixels.

Figure 2: (A) Signal composed of 2 sinusoidal modes: a slow one (around $20 \mathrm{~Hz}$ ), and a faster one (around $35 \mathrm{~Hz}$ ) each of them non constant in frequency; (B) rescaled scalogram obtained with Morlet wavelet $\left(\omega_{0}=12\right)$ and coded with 64 gray levels from black $\min (P(x, a))$ to white $(\max (P(x, a))$. The black lines correspond to the location of the ridges obtained. (C, D) Phases extracted (solid line) from low frequency and high frequency signals respectively using the wavelet ridge method (theoretical phase is indicated by the dashed line). (E, F) Same as (C) and (D) but obtained with Hilbert transform of the signal filtered between $10-28 \mathrm{~Hz}$ and $28-50 \mathrm{~Hz}$.

Figure 3: Bias and variance of the method as a function of SNR. (A) Normalized bias of the estimated frequency. (B) Standard deviation of the estimated frequency. (C) Bias for the estimated phase. (D) Standard deviation for the estimated phase. Bias and variance are computed on signals used in Fig.2 in 2 different frequency bands: * for high frequency, ${ }^{\circ}$ for low frequency. 
Figure 4: Limit of the wavelet ridges method: (A) Raw signal composed of 2 sinusoidal modes crossing each other in frequency $(\mathrm{t}=2 \mathrm{sec})$. (B) Scalogram with superimposed wavelet ridge (solid line).

Figure 5: Preliminary tests setting the right wavelet shape for wavelet ridge extraction. (A) Raw signal composed of 2 closed sinusoidal modes non constant in frequency. (B-C-D): Scalograms computed with increasing wavelet coefficient $\left(\omega_{0}=6,12,24\right)$. This parameter should be set carefully to allow accurate extraction of the wavelet ridge (C).

Figure 6: Analysis of an olfactory bulb activity. (A) Single trial, whole raw signal, duration 15 sec. (B) Corresponding low time-resolution scalogram. The white thick line at bottom indicates the 5 seconds long odor stimulation. Total number of spikes: $n=71$. (C) Enlargement of inset in (A) showing both LFPs oscillations and spiking activity for a 1 second duration. (D) Corresponding low time-resolution scalogram. The thick black lines represent the wavelet ridges obtained from the high resolution scalogram. (E) Linear phase extracted from each wavelet ridge. (F, G) SPIPHs constructed from $\beta(F)$ and $\gamma(G)$ episodes. Solid line: LFPs phase. Histograms were computed from the 5-sec odor evoked response (SPIPH for $\beta$ (F) $\mathrm{n}=18$ spikes; SPIPH for $\gamma(\mathrm{G}): \mathrm{n}=48$ spikes).

Figure 7: Analysis of a piriform cortex activity. (A) Low resolution scalogram (top) and corresponding raw signal (bottom) showing both LFPs oscillations in the $\gamma$ range and a spiking unitary activity (total number of spikes: n=73). (B) Enlargement of the inset in A. From top to bottom: low resolution scalogram displaying local maxima $(*)$ and wavelet ridge (white solid line); raw signal; phase extracted along the ridge with superimposed location of spikes (open circle). (C) SPIPH during odor stimulation ( $5 \mathrm{sec}$ ) computed from the single cell 
shown in A, B ( $\mathrm{n}=21$ spikes). (D) SPIPH during odor stimulation $(5 \mathrm{sec})$ computed from a repetition of six trials $(\mathrm{n}=314$; mean phase $=-3.00 \pm 1.13 \mathrm{rad})$. 

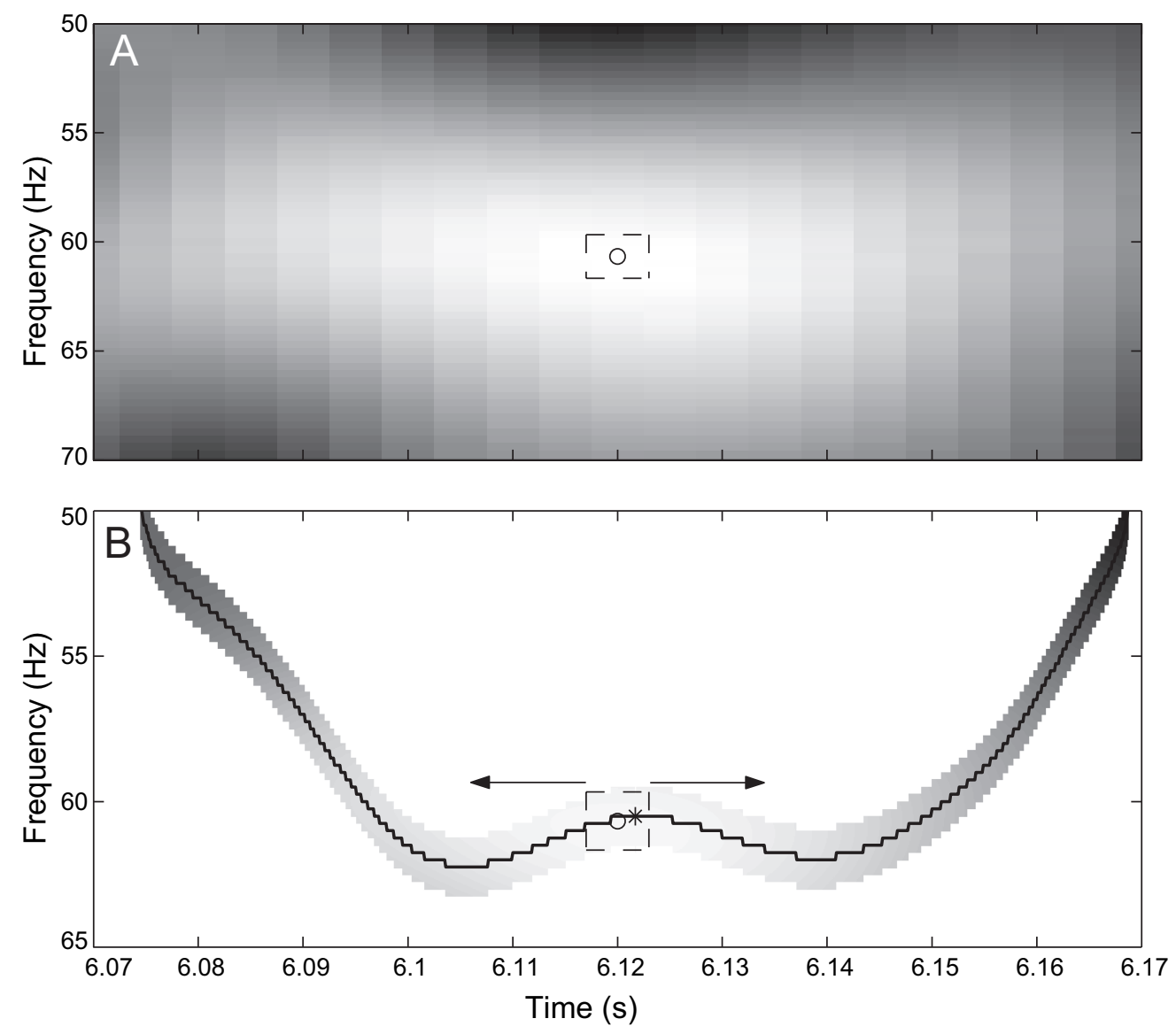

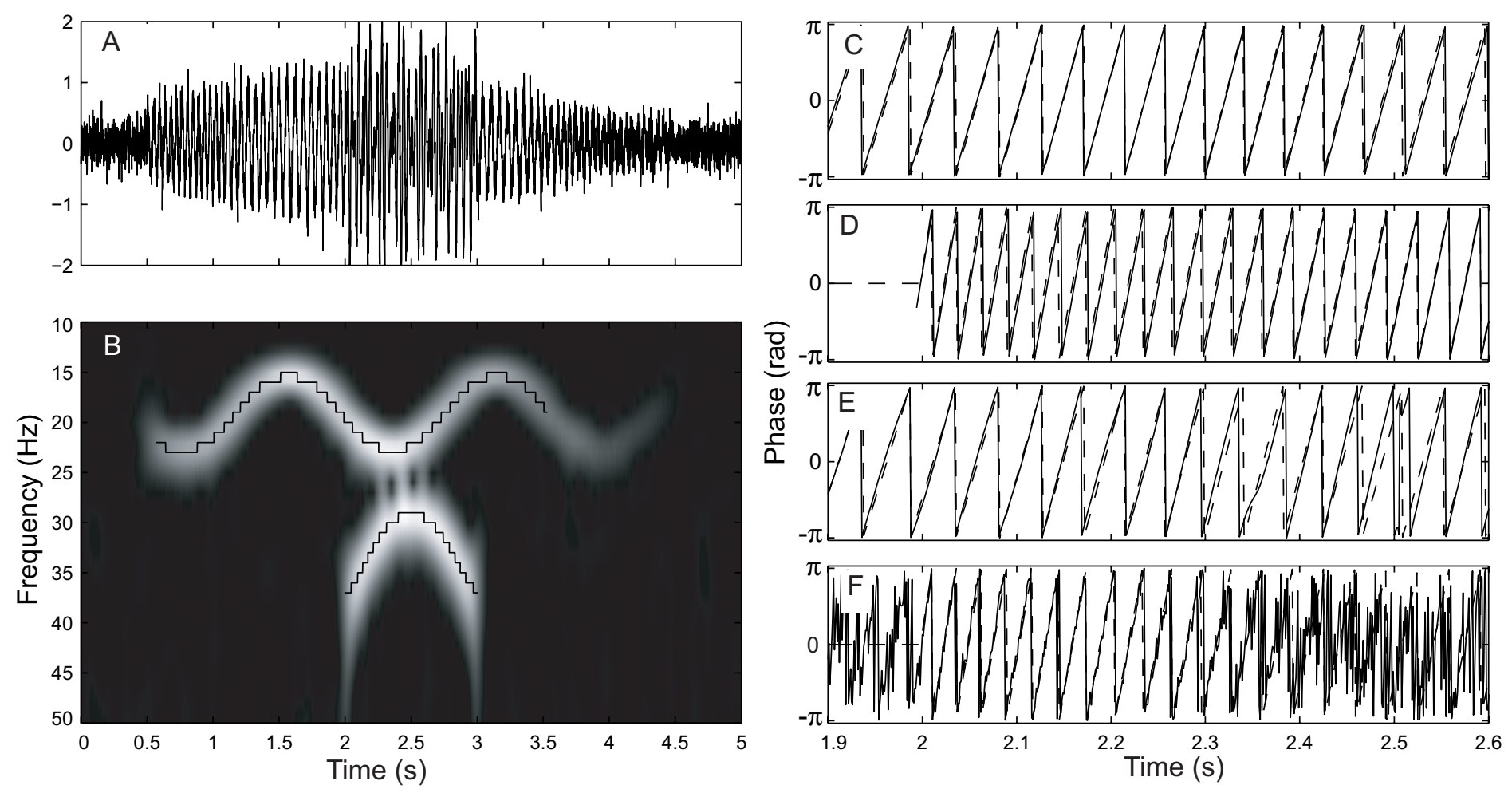

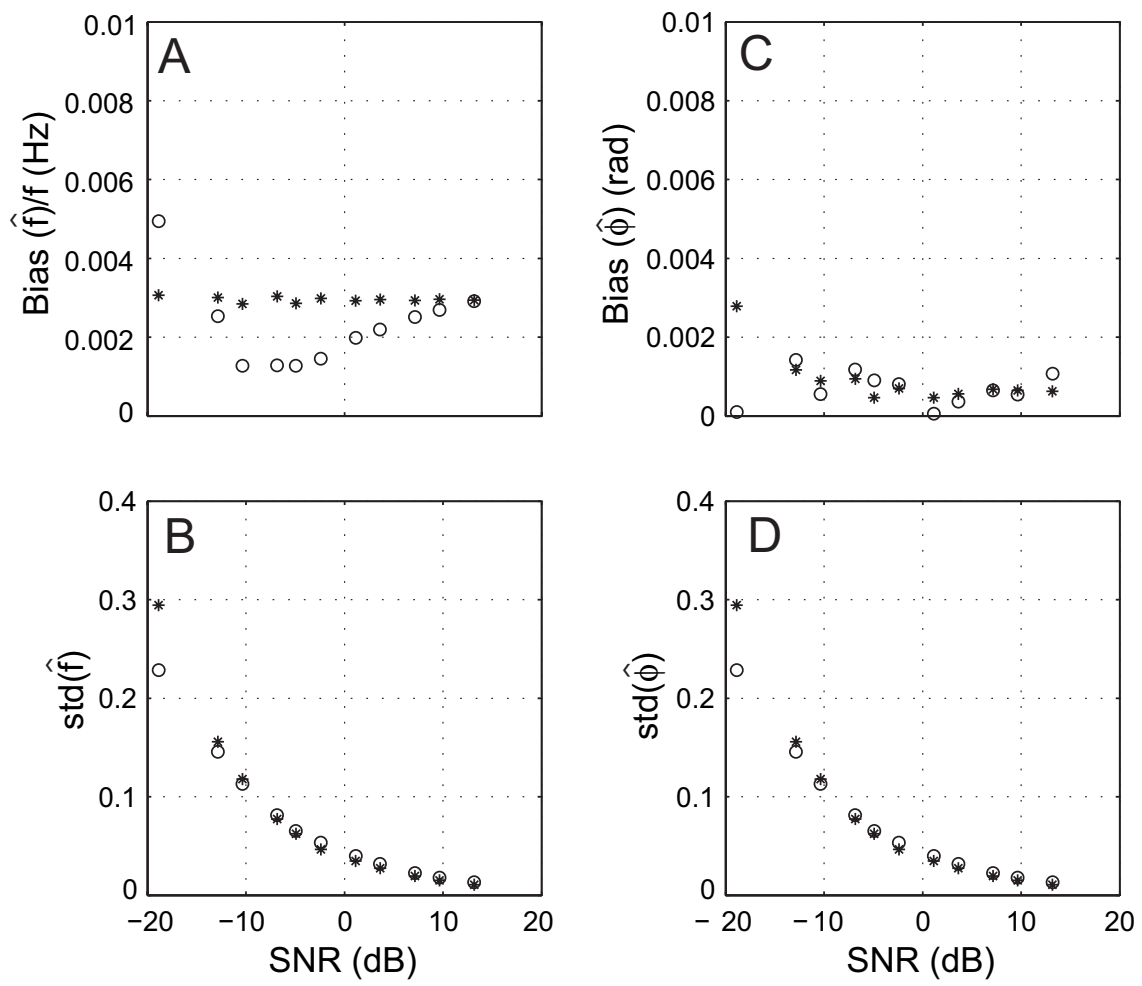

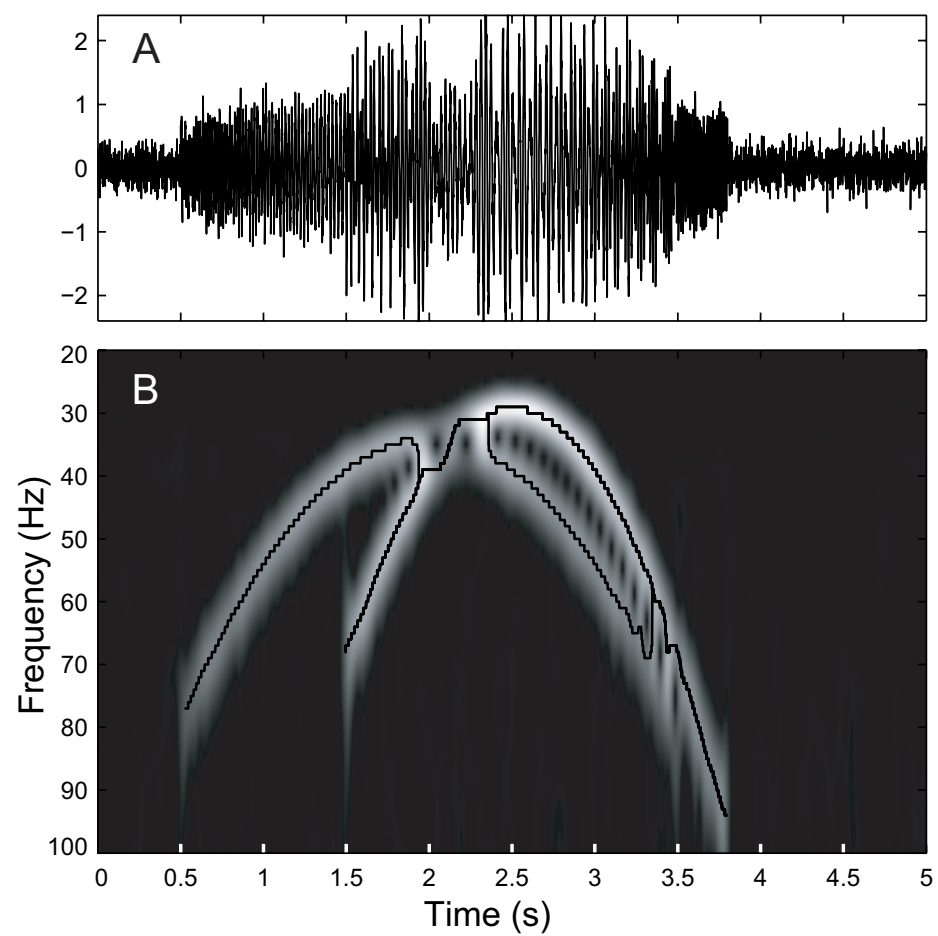

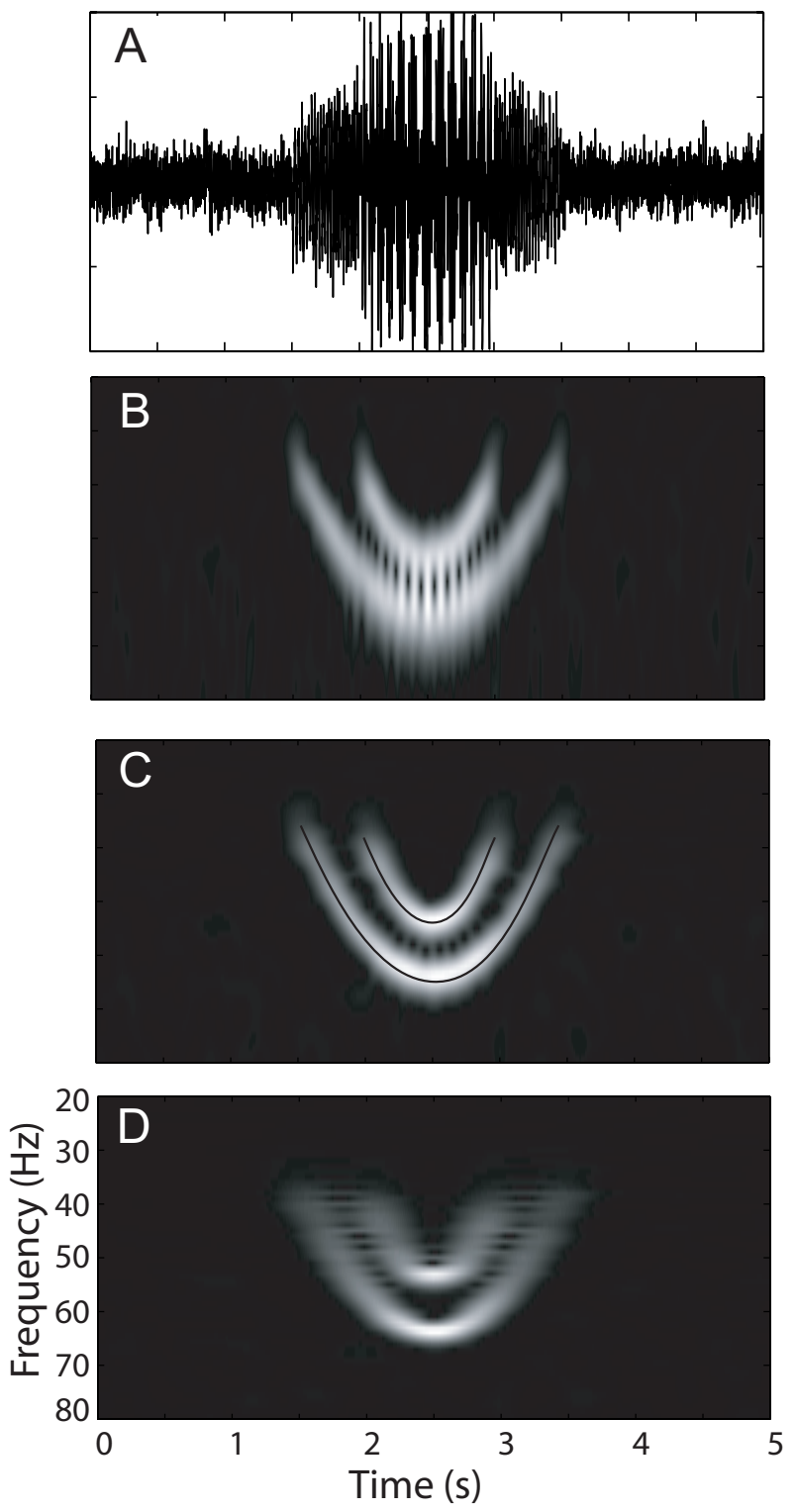

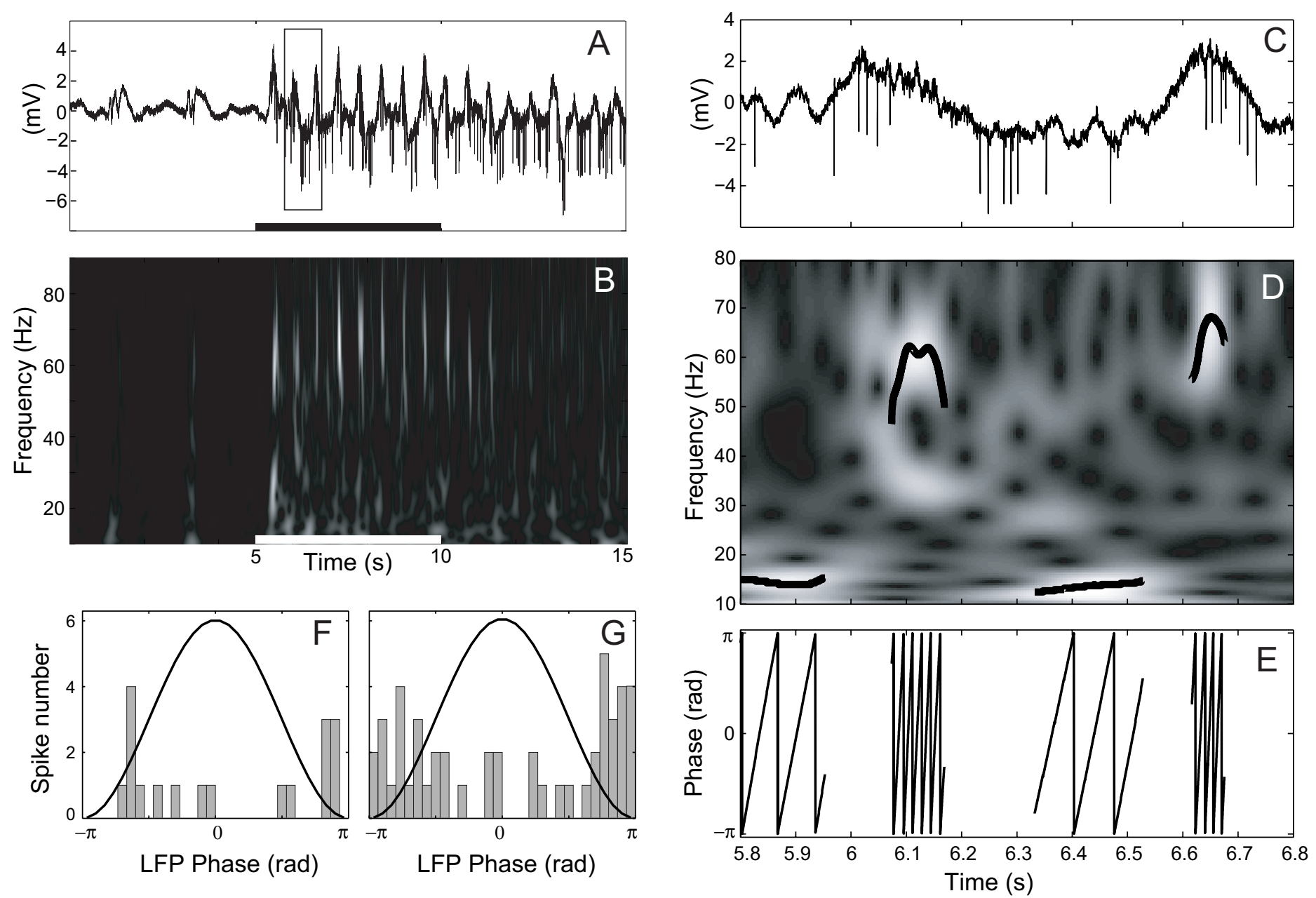

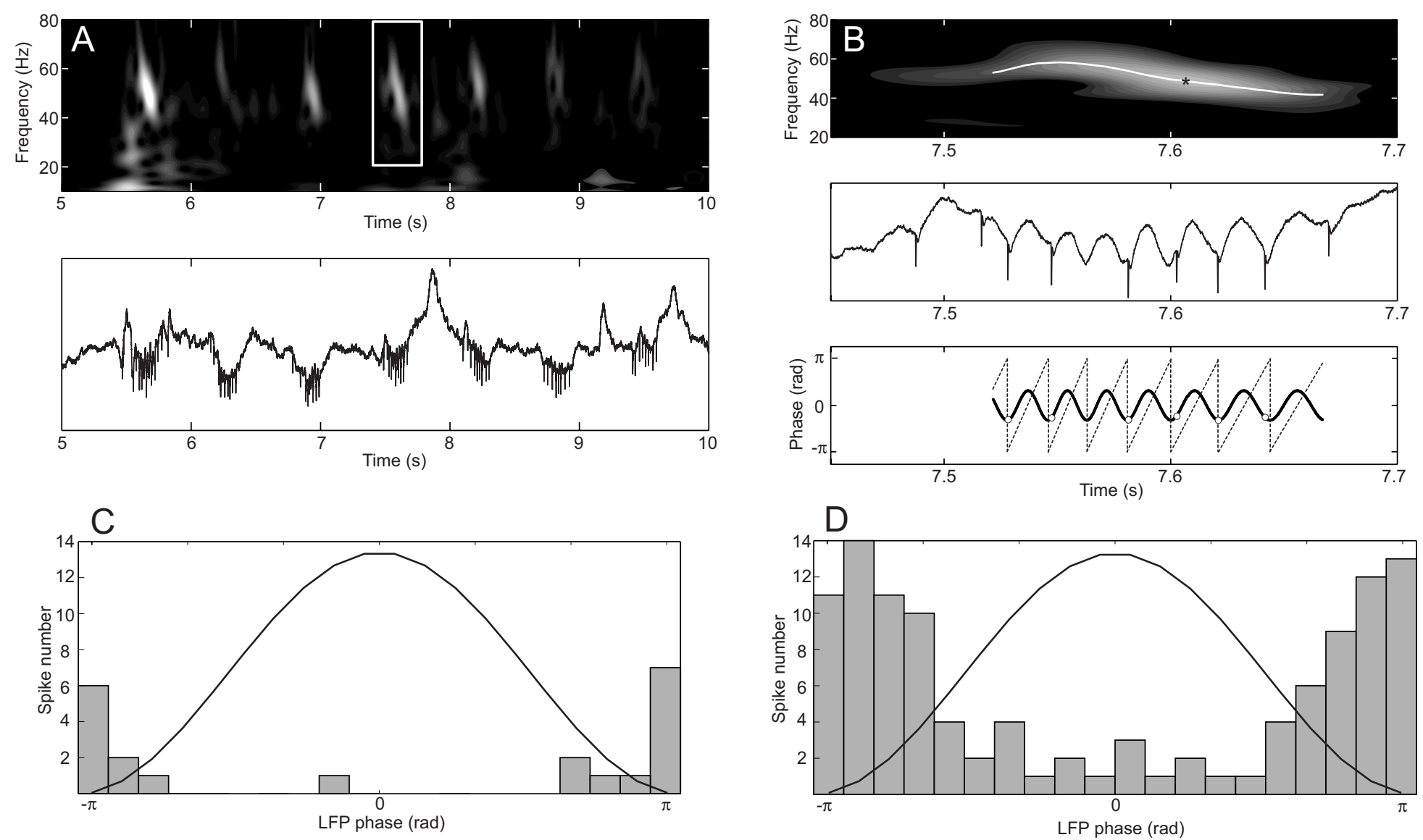